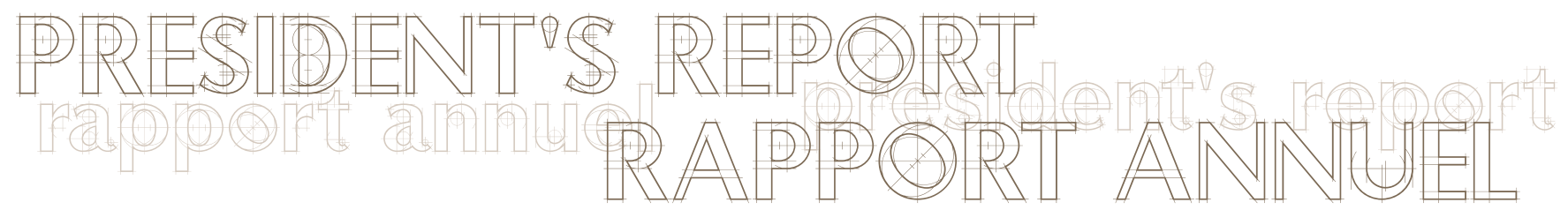

\title{
CAO President's Annual Report
}

\section{Rapport annuel du président de I'ACO}

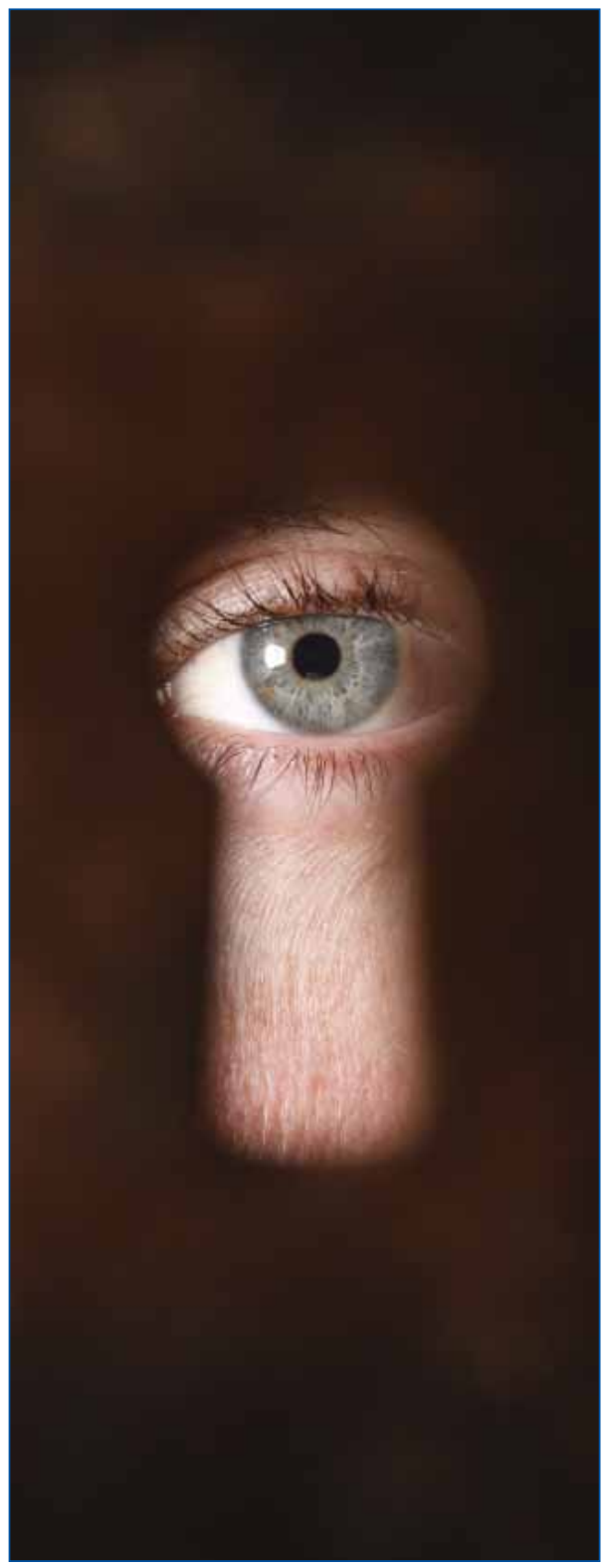

I am very pleased to report on CAO's activities for this past year July 2007 - July 2008. Firstly, I must say how quickly the time has passed! It seems like only a very few short weeks ago that we were at Congress in Saskatoon, and then the Alberta Annual General Meeting in Calgary distributing hats and ' $T$ ' shirts with excitement and anticipation for a great year ahead. Much happened since then and I believe we have made progress in many areas, which are summarized as follows:

\section{(1) Implementing the CAO Strategic Plan} and Action plan. At our first Council meeting in Calgary we confirmed our intention to follow through with the strategic directions recommended for 2006 - 2009. Our actions and successes in 2007 - 2008 have been that:

- We have influenced government policy by successfully obtaining reclassification for all contact lenses including 'cosmetic' lenses as medical devices as well as recognition of optometrists as 'prescribers' under Bill C51. We have held meetings with representatives of the Health Ministry (March 7th), Progressive Licensing Department (April 30th), with PHAC, (May 7th), and the Competition bureau (May 16th).

- We are elevating the Standard of care through a new Diabetes Committee, its core document, and making huge strides towards implementation of TPA legislation in Ontario, Manitoba, Prince Edward Island and British Columbia.

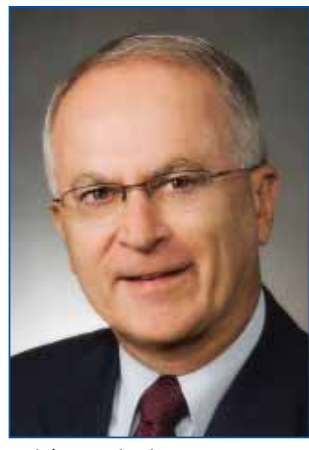

With optimism and commitment for 2008-2009,

Respectfully submitted, Len Koltun, OD

President CAO

C'est avec optimisme et résolution pour 2008-2009 que je vous présente espectueusement ce rapport.

Len Koltun, OD

Président de I'ACO 


\section{PRESIDENT'S REPORT RAPPORT ANNUEL}

We are improving and emphasizing communications in many areas: inter, intra professional, with students and the public. At the first Council meeting of my term, CAO council supported my suggested President's theme of 'Communication is the Key' - by promoting communications we will be able to open many doors for optometry. We have followed through with this theme in many areas:

- M ember communications has improved through our revised member website platform design, a new In Touch format and completing a membership satisfaction survey in M ay 2008.

- We are reaching out and attempting to communicate to all $\mathrm{N}$ orth A merican optometry students by offering them a free membership.

- Both the new diabetes initiative (D r. Richard L $œ$ ) and CA 0 's 0 ccupational V ision Program are re emphasizing communications as the theme to educate and inform the public of optometry's role in the management of people with diabe tes and the importance of eye safety.
- Perhaps our greatest achievement this year was that the Canadian Public Relations Society awarded a Silver M edal Recognition A ward to N PE C, our N ational Public E ducation Program, for 'Best $\mathrm{U}$ se of $\mathrm{C}$ ommunications Tools' for the $2007 \mathrm{E}$ ye $\mathrm{H}$ ealth $\mathrm{M}$ onth Campaign. This is an outstanding tribute to the dedication and commitment of $\mathrm{C}$ hair D r. L il L inton, her committee and all C anadian optometrists who have faithfully supported this initiative, which has become the model and the envy of other professional organizations. W e can be ex tremely proud of this acomplishment. - Through 'Presidents Travels' summaries I have attempted to keep CA 0 C ouncil aware and up to date on activities and priorities of all CA 0 orporate members. I also look forward to writing the President's Podium in the $\mathrm{C}$ anadian Journal of 0 ptometry, relaying updates on CA 0 activities and thoughts about the future of optometry.

- We have revised CAO infrastructure to achieve our goals with office renovations now completed; hired

\section{The key benefits of group insurance from The Personal}

Preferred Rates
Only available to
members of the
Canadian Association
of Optometrists




\section{RAPPORT ANNUEL PRESIDENT'S REPORT}

a Webmaster, and adopted a new Council and Executive meeting format that reflects our strategic directions.

- We adopted a new template to monitor progress of the strategic plan. It is an 'organic' document, reviewed at all our council meetings and serves as an efficient blueprint to track strategies, actions and progress.

(2) Implementing the 2007 0ptometric Leaders' Forum (OLF) Futures Recommendations. The OLF Futures recommendation of 1) increased recognition, improved integration, 3) elevating the Standard of Care, 4) improving government relations and, 5) improving individual $\mathrm{OD}$ awareness were a reaffirmation of the CAO Strategic Plan. This validation has increased the importance of striving to achieve the goals as set out in the Strategic Plan.

(3) Obtaining TPAs for all Canada is nearly a reality with ON, MB, PEI and BC. We expect to see TPA legislation implemented throughout Canada this next year.

(4) Funding on new research projects. CAO provided funding for, 1) a Forum discussion at the School of Optometry, UW to describe the profile of a 2015-optometry graduate, and 2) a joint study by COETF and CNIB to determine the incidence of uncorrected refractive error in the Canadian population.

5 Public Visual Welfare Committee This committee continues to monitor policy and legislation to ensure a comprehensive standard of eye care in Canada. The provision of 'sight tests' by opticians remains in place in BC, Alberta and Ontario, despite questionable legal standing. CAO is monitoring outcomes of 2 court proceedings: 1) an appeal by an Ontario optician who was found guilty of prescribing from sight testing measurements, and 2) Quebec decision that opticians rescind certain sections of their bylaws and that they 'cease to perform acts that fall solely under the practice of optometry'... like over refraction and 'eye examinations'.

(6) This fall we are embarking on an improved CAO OVP program (Ontario) with emphasis on communications to expand the program and increase awareness with industry.
(7) Providing speakers. We are pleased that Dr. Chris Hudson will be speaking to the 2008 Family Physician's Forum in Toronto and Dr. Langis Michaud to the 2008 CDA forum in Montreal.

8 Expanding Member Programs. CAO Council approved an expansion to the Centennial private label frames sales program to include several 'higher end' models and will launch a new merchant card program with Chase paymentech.

(9) Continued assistance for COETF and OGS. $\mathrm{CAO}$ provides ongoing secretariat support for both these worthwhile optometric charities.

(11) Maintaining responsible governance. We are focused on operational excellence and committed to delivering advocacy for optometry. The entire CAO Council, and staff are to be congratulated for administering association affairs with due diligence committed to success. Thank You!! Our strategy of financial discipline and investing in our infrastructure is providing the support needed to achieve our goals.

\section{Moving forward}

This past year has stimulated my passion. desire and commitment to advance optometry further. The year has gone by so quickly I feel I have taken only the first step in establishing many key relationships for optometry and CAO. I believe I can cultivate these further as well as create others.

Another reason why this coming year and a second term is so special and important is that I believe we have a clear message and a clear goal to continue the momentum to complete what we've just started. I believe we have made significant progress and I would like to thank everyone for their support, and in particular CAO Council for their endorsement for a second term.

I have great confidence in Optometry's future. I continue to believe in the dream that optometry have the recognition and respect that it deserves, and I continue to believe that the theme 'Communication is the Key' is an important element of our future success- that improved member communication will make us a stronger association and improved public communication will educate and elevate optometry's profile and status.

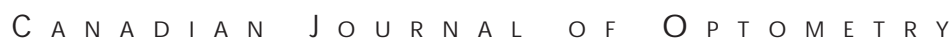

R E V U E C A N A D I E N N E D ' O P T O M É T R I E
Vol 70 No 5

September / septembre 2008 


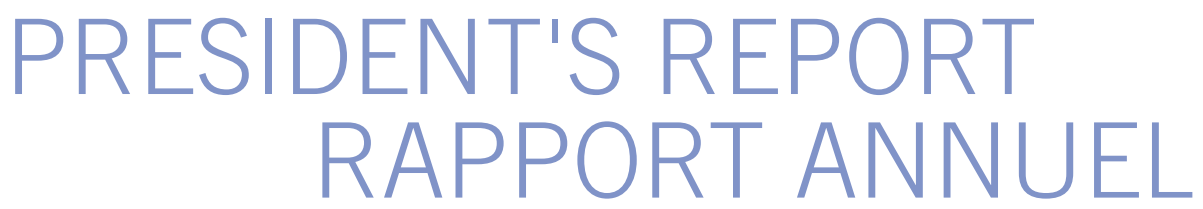

J e suis heureux de rendre compte des activités de l'ACO pendant la dernière année, soit de juillet 2007 à juillet 2008. Je dirai d'abord que le temps a passé si vite! Il me semble que, il y a seulement quelques courtes semaines, nous étions au congrès à Saskatoon. Beaucoup d'eau a coulé sous les ponts depuis et, à mon avis, nous avons progressé dans bien des secteurs. Je résumerai ces progrès :

\section{(1) Mise en application du plan stratégique et du} plan d'action de l'ACO. À la première réunion du Conseil à Calgary, nous avons confirmé notre intention de réaliser les orientations stratégiques recommandées pour 2006-2009. Nos activités et nos réalisations en 2007-2008 ont été les suivantes :

- Nous avons influencé la politique du gouvernement en obtenant le reclassement de toutes les lentilles cornéennes, y compris les lentilles « cosmétiques », à titre de matériel médical, ainsi que la reconnaissance des optométristes comme "prescripteurs » dans le projet de loi C51. Nous avons eu des réunions avec les représentants du ministère de la Santé (7 mars), du Projet d'homologation progressive (30 avril), de l'ASPC (7 mai) et du Bureau de la concurrence (16 mai).

- Nous élevons les normes de soins grâce à un nouveau comité du diabète et son document de base et nous faisons de très grands pas vers l'adoption de mesures législatives relatives aux APT en Ontario, au Manitoba, à l'Île-du-Prince-Édouard et en C.-B.

- Nous améliorons et valorisons nos communications dans bien des secteurs, soit à l'échelle interprofessionnelle et intraprofessionnelle et avec les étudiants et le public. À la première réunion du Conseil pendant mon mandat, celui-ci a retenu le thème que je proposais «La communication est la clé ». En favorisant les communications, nous pourrons ouvrir bien des portes à l'optométrie. Nous avons cultivé ce thème dans nombre de secteurs :

- L es communications avec les membres se sont améliorées grâœà une reconception dela plateforme du site W eb des membres, à une nouvelle présentation de C ontact et à un sondage sur la satisfaction de ces mêmes membres en mai 2008.

- $\mathrm{N}$ ous tendons la main à tous les étudiants nord-américains en optométrie et tentons d'entrer en communication avec eux en

leur offrant l'adhésion gratuite à notre association.

- D ans la nouvelle initiative portant sur le diabète (Dr Richard L e) comme dans le Programme professionnel des soins de la vue, nous insistons à nouveau sur les communications comme le thème d'une éducation et d'une information du public sur le rôle de l'optométrie dans la prise en charge des diabétiques, ainsi que sur l'importance de la sécurité oculaire. - Peut-être notre plus grande réalisation œette année a-t-elle été l'octroi par la Société canadienne des relations publiques d'une médaille d'argent au CNE P - notre Programme national d'éducation publique - dans la catégorie « M eilleure utilisation d'outils de communications » dans le cas de la campagne du M ois de la santé de l'œil 2007. C'est là une reconnaissance insigne du travail inlassable et empressé de la présidente, la D re L il L inton, de son comité et de tous les optométristes canadiens qui ont sans cesse appuyé cette initiative devenue l'envie et le modèle pour les autres organismes professionnels. N ous ne pouvons qu'être ex trêmement heureux de ctte réalisation.

- Par des résumés des déplacements du président, j'ai essayé de tenir le Conseil de l'A CO au fait des activités et des priorités de toutes les sociétés membres de l'A CO. J'ai également hâte d'écrire le Mot du président dans la Revue canadienne d'optométrie; j'y ferai le point sur les activités de l'A CO et livrerai mes réflex ions sur l'avenir de l'optométrie.

- Nous avons révisé l'infrastructure de l'ACO en vue de la réalisation de nos objectifs. Les travaux de rénovation des bureaux ont été menés à bien. Nous avons embauché un webmestre et adopté pour les séances du Conseil et du Comité exécutif une nouvelle formule qui traduit nos orientations stratégiques.

- Nous avons officialisé un nouveau modèle de surveillance des progrès dans l'exécution du plan stratégique. Il s'agit là d'un document « organique » qui est révisé à toutes les réunions du Conseil et sert de plan pratique de suivi des stratégies, des mesures et des progrès.

(2) Mise en application des recommandations du Sommet de l'avenir de 2007 du Forum des dinigeants optométriques. Que le Sommet sur l'avenir du FDO ait recommandé 1) d'accroître la reconnaissance de l’optométrie, 2) d'améliorer l'intégration, 3) d'élever les normes de soins, 4) de resserrer les liens avec le gouvernement et 5) de sensibiliser individuellement les OD n'est qu'une réaffirmation de ce que dit le Plan straté- 


\section{RAPPORT ANNUEL PRESIDENT'S REPORT}

gique de l'ACO. Cette confirmation confère encore plus d'importance à nos efforts en vue d'atteindre les objectifs de ce plan.

\section{(3) La mise en place d'un cadre législatif des APT} partout au pays est presque devenue réalité en Ontario, au Manitoba, à l'Île-du-Prince-Édouard et en ColombieBritannique. Nous nous attendons à ce que, au cours de l'année qui vient, des mesures législatives soient mises en place dans tout le Canada.

(4) Financement de nouveaux projets de recherche. L'ACO a financé 1) un débat à l'École d'optométrie de l'Université de Waterloo en vue de faire le portrait du diplômé de 2015 en optométrie et 2) une étude coréalisée par le FFOCE et l'INCA sur la fréquence des erreurs réfractives non corrigées dans la population canadienne.

5 Comité sur le bien-être visuel du public. Ce comité continue à surveiller les politiques et les lois afin de garantir l'application de normes d'ensemble sur les soins oculo-visuels au Canada. En Colombie-Britannique, en Alberta et en Ontario, les opticiens s'occupent toujours d'examens de la vue, bien que cette pratique soit juridiquement contestable. L'ACO surveille l'aboutissement de deux causes en justice, à savoir 1) l'appel interjeté par un opticien de l'Ontario reconnu coupable d'avoir prescrit à partir de mesures de «test de la vision » et 2) une décision en appel des tribunaux québécois ordonnant aux opticiens d'annuler certaines dispositions de leur règlement et de cesser d'exécuter des actes qui relèvent exclusivement de la pratique optométrique (surréfraction, examens de l'œil, etc.).

(6) Cet automne, nous lançons pour le régime professionnel des soins de la vue un programme amélioré avec l'Ontario où nous mettrons l'accent sur les communications en vue d'étendre les activités et de mieux sensibiliser l'industrie.

(7) Service de conférenciers. Nous sommes heureux d'annoncer que les Drs Chris Hudson et Langis Michaud prendront respectivement la parole en 2008 devant le Forum en médecine familiale à Toronto et le forum de l'ACD à Montréal.

8 Extension des programmes aux membres. Le
Conseil de l'ACO a approuvé l'extension à plusieurs modèles « haut de gamme » du programme des montures « sous marque du fabricant » de Centennial. Il lancera un nouveau programme de cartes de commerçants avec Chase Paymentech.

\section{(9) Maintien de l'aide au FFOCE et à l'« Optometry} Giving Sight ». L'ACO assure en permanence des services de secrétariat à ces deux louables organismes de bienfaisance de l'optométrie.

(II) Maintien d'une gouvernance en toute responsabilité. Nous nous vouons à l'excellence opérationnelle et à la promotion de l'optométrie. Nous félicitons tous les conseillers et les membres du personnel d'administrer les affaires de l'Association avec diligence dans un souci de réussite. Merci à tous! Notre stratégie de discipline financière et d'investissement dans notre infrastructure constitue le point d'appui dont nous avons besoin pour atteindre nos objectifs.

\section{Prochaines étapes...}

Cette dernière année a stimulé mon désir passionné et ma résolution de faire progresser l'optométrie. Le temps a passé si vite que j'ai l'impression d'avoir seulement effleuré les possibilités d'apprentissage et de n'avoir fait qu'un premier pas dans l'établissement de tout un réseau de relations clés pour l'optométrie et l'ACO. Je crois pouvoir cultiver ces relations et en susciter d'autres.

Une autre raison pour laquelle l'année qui vient et un second mandat sont si spéciaux et importants est que, à mon avis, le message et le but sont clairs : il nous faut continuer et mener à bien ce que nous avons si bien commencé. À mes yeux, les progrès sont nets, et je remercie tout le monde de son appui et, en particulier, le Conseil de l'ACO d'avoir approuvé un autre mandat.

J'ai toute confiance dans l'avenir de l'optométrie. J'ai toujours ce rêve d'une optométrie jouissant de la reconnaissance et du respect qu'elle mérite. Pour moi, le thème « La communication est la clé » est un grand facteur de réussite pour demain. L'amélioration des communications avec les membres nous renforcera comme association et, si nous faisons de même avec le public, l'image et le statut de l'optométrie seront plus connus et rehaussés d'autant.

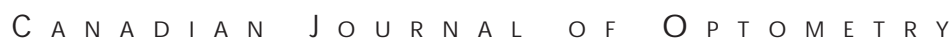

R E V U E C A N A D I E N N E D ' O P T O M É T R I E
Vol 70 No 5

September / septembre 2008 